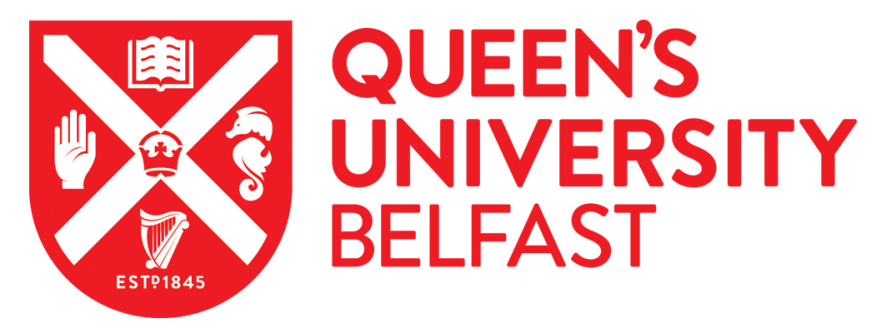

\title{
Thermodynamic properties and atomistic structure of the dry amorphous silica surface from a reactive force field model
}

Cabriolu, R., \& Ballone, P. (2010). Thermodynamic properties and atomistic structure of the dry amorphous silica surface from a reactive force field model. Physical Review B (Condensed Matter), 81(15), [155432]. https://doi.org/10.1103/PhysRevB.81.155432

Published in:

Physical Review B (Condensed Matter)

Document Version:

Publisher's PDF, also known as Version of record

Queen's University Belfast - Research Portal:

Link to publication record in Queen's University Belfast Research Portal

Publisher rights

Copyright 2010 American Physical Society.

This work is made available online in accordance with the publisher's policies. Please refer to any applicable terms of use of the publisher.

\section{General rights}

Copyright for the publications made accessible via the Queen's University Belfast Research Portal is retained by the author(s) and / or other copyright owners and it is a condition of accessing these publications that users recognise and abide by the legal requirements associated with these rights.

Take down policy

The Research Portal is Queen's institutional repository that provides access to Queen's research output. Every effort has been made to ensure that content in the Research Portal does not infringe any person's rights, or applicable UK laws. If you discover content in the Research Portal that you believe breaches copyright or violates any law, please contact openaccess@qub.ac.uk. 


\title{
Thermodynamic properties and atomistic structure of the dry amorphous silica surface from a reactive force field model
}

\author{
Raffaela Cabriolu ${ }^{1}$ and Pietro Ballone ${ }^{2}$ \\ ${ }^{1}$ Centre for Molecular Nanoscience, University of Leeds, Leeds LS2 9JT, United Kingdom \\ ${ }^{2}$ Atomistic Simulation Centre, Queen's University Belfast, Belfast BT7 1NN, United Kingdom \\ (Received 17 January 2010; revised manuscript received 20 March 2010; published 16 April 2010)
}

\begin{abstract}
A force field model of the Keating type supplemented by rules to break, form, and interchange bonds is applied to investigate thermodynamic and structural properties of the amorphous $\mathrm{SiO}_{2}$ surface. A simulated quench from the liquid phase has been carried out for a silica sample made of 3888 silicon and 7776 oxygen atoms arranged on a slab $\sim 40 \AA$ thick, periodically repeated along two directions. The quench results into an amorphous sample, exposing two parallel square surfaces of $\sim 42 \mathrm{~nm}^{2}$ area each. Thermal averages computed during the quench allow us to determine the surface thermodynamic properties as a function of temperature. The surface tension turns out to be $\gamma=310 \pm 20 \mathrm{erg} / \mathrm{cm}^{2}$ at room temperature and $\gamma=270 \pm 30$ at $T=2000 \mathrm{~K}$, in fair agreement with available experimental estimates. The entropy contribution $T s_{s}$ to the surface tension is relatively low at all temperatures, representing at most $\sim 20 \%$ of the surface energy. Almost without exceptions, $\mathrm{Si}$ atoms are fourfold coordinated and oxygen atoms are twofold coordinated. Twofold and threefold rings appear only at low concentration and are preferentially found in proximity of the surface. Above the glass temperature $T_{g}=1660 \pm 50 \mathrm{~K}$, the mobility of surface atoms is, as expected, slightly higher than that of bulk atoms. The computation of the height-height correlation function shows that the silica surface is rough in the equilibrium and undercooled liquid phase, becoming smooth below the glass temperature $T_{g}$.
\end{abstract}

DOI: $10.1103 /$ PhysRevB.81.155432

PACS number(s): 68.35.bj, 65.60.+a, 68.35.Ct, 68.35.Md

\section{INTRODUCTION}

Silicon dioxide $\mathrm{SiO}_{2}$ (silica) is one of the most important minerals, ${ }^{1}$ present in nature as quartz, cristobalite, and as an amorphous solid, while exotic phases such as coesite and stishovite can be found in meteor craters. More importantly, silica represents the backbone of an endless number of silicates. ${ }^{2}$ Silica is also one of the oldest known technological materials, used since the beginning of civilization and still represents an essential component in state-of-the-art electronic devices. It comes as a surprise, therefore, to realize that despite the simplicity of its constituents and despite the vast number of experimental and theoretical studies, so much is still unknown for this material. This is particularly true of its surface, whose complexity has until now prevented a full understanding of its properties. ${ }^{3}$ This state of affair is shared with the wider class of oxide surfaces, ${ }^{4}$ whose structure, precise composition, surface charge, and reactivity depend sensitively on preparation, ambient chemical conditions, and surface treatment.

The dependence of the surface properties of silica on preparation manifests itself, for instance, in the different hydrophobicity of seemingly equivalent samples. Freshly cleaved silica surfaces are reactive and in the presence of water give rise to silanols, consisting of hydroxyl groups bonded to $\mathrm{Si}$ atoms by covalent and ionic forces. The dangling $\mathrm{OH}$ groups of silanols impart a degree of affinity for water, turning the hydrophobic surface into an hydrophilic one at sufficiently high density of $\mathrm{OH}$ groups. ${ }^{5}$

Dry silica surfaces can be recovered by thermal treatment, i.e., by annealing at $T \geq 650 \mathrm{~K}$ for at least a few hours, and measurements show that these surfaces tend to be hydrophobic, ${ }^{6}$ remaining so for macroscopic times even after being exposed to air at ambient conditions. Although the dry surface obtained in this way is, arguably, the simplest termination of silica solids, several among its properties are still far from being understood, ${ }^{3}$ especially in the amorphous case. To be precise, many experimental studies have been devoted to amorphous silica surfaces, whose detailed characterization, however, is hampered by experimental difficulties. Powerful structural techniques such as low-energy electron diffraction (LEED), relying on crystal-like two-dimensional (2D) periodicity, cannot be used in this case. Moreover, the insulating character of silica represents another difficulty, since spectroscopic techniques such as scanning tunneling microscopy (STM), electron energy loss (EELS), and, to some extent, x-ray photo-electron spectroscopy (XPS) require conducting, electrically grounded samples to provide highly reproducible data. Finally, in the case of the amorphous surface, a direct connection between observed properties and an atomistic interpretation is made difficult by the nonuniqueness of the surface structure that admits only a statistical description.

Despite these limitations, a few basic facts are widely accepted by the surface science community. For instance, it is generally agreed that the response of the silica surface to $\mathrm{x}$-ray photoemission measurements is dominated by the oxygen signal, ${ }^{7}$ implying that this is the atom type most likely to intercept incoming photons and thus suggesting that oxygen occupies the outermost surface layer. Undercoordinated oxygen atoms, representing the so-called nonbridging oxygens (NBOs), are not detected by electron-spin resonance (ESR, see Ref. 8) and by core-level photoelectron spectroscopy ${ }^{9}$ on dry, annealed silica surfaces, while both NBO and silicon coordination defects ( $E^{\prime}$ centers) are found on amorphous surfaces freshly produced by sputtering or irradiated at UHV conditions. ${ }^{10}$ The surface tension is difficult to measure quantitatively for all solid surfaces, but the most reliable es- 
timates for amorphous silica range around $\gamma \sim 300 \mathrm{erg} / \mathrm{cm}^{2}$ (see Ref. 11).

Additional information on structure and bonding is provided by optical (Raman and infrared ${ }^{12}$ ) and NMR (Ref. 13) spectroscopies. Adsorption isotherms for a variety of molecules are also available (see, for instance, data for $\mathrm{Ar}$ and $\mathrm{CO}_{2}$ in Refs. 14 and 15, respectively). The information provided by these measurements is only indirect and requires modeling ${ }^{16,17}$ to be translated into microscopic understanding.

Theoretical and computational investigations of silica surfaces have been represented primarily by $a b$ initio determinations of the atomic and electronic structures of ordered surfaces, ${ }^{18-20}$ by force field investigations of surface vibrational properties, and by computer simulations of ordered and especially amorphous surfaces at nonzero temperature, based on empirical model potentials. Mixed quantum mechanical-molecular mechanics (QMMM) approaches have been used as well to investigate the grafting of organic molecules on the surface of dry amorphous silica. ${ }^{21}$

Pair- and few-body potentials, describing silica as a predominantly ionic material and able to reproduce a fairly wide variety of its properties, have been developed by several groups and are defined and discussed in Refs. 22-29. This last, in particular, including two- and three-body terms, arguably is the most thoroughly tested among this family of models, even though in the last few years, the potential of Ref. 28 has also been used extensively. Fluctuating charge models ${ }^{30}$ and polarizable ion potentials, ${ }^{31}$ which are intrinsically many-body, could provide a significant improvement with respect to few-body potentials once parametrized against $a b$ initio and/or spectroscopy data. Recently, a model able to describe ordered and amorphous silica surfaces in contact with water has been proposed in Ref. 32 .

The application of all these models to the investigation of the amorphous silica surface has produced a wealth of microscopic information on the local structure and atomic dynamics in these systems. An exhaustive review is beyond the scope of the present introductory section. Representative molecular-dynamics simulations of the amorphous silica surface include the studies presented in Refs. 17 and 33-35. In the last of these studies, molecular dynamics based on a classical (ionic) potential including electron polarization has been combined with $a b$ initio methods for silica clusters terminated by hydrogens or $\mathrm{OH}$ groups to elucidate the electronic properties of representative structural motifs.

In most of the simulation studies mentioned above, the amorphous surface is obtained by quenching a liquid slab using molecular dynamics. The relatively short simulation time results in very fast quenches, often giving rise to a concentration of bonding defects and especially of NBOs higher than measured in experiments (see the discussion in Ref. 36). In the case of pair potentials, in particular, the surface energy and surface tension tend to be significantly higher than experimental estimates ${ }^{34}$ and, especially with early silica models, ${ }^{23,25}$ an unphysical population of overcoordinated silicon and oxygen atoms is observed. A few molecular-dynamics simulations of silica surfaces have also been performed based on $a b$ initio methods. ${ }^{18,19,37,38}$ The time scale accessible to ab initio methods is 2 or 3 orders of magnitude shorter than for classical potentials and also the size scale is severely restricted, thus limiting the ability of $a b$ initio simulation to represent amorphous surfaces. These problems, of course, are to some extent compensated by the fact that $a b$ initio models provide a comprehensive description of ionic and covalent bondings, as well as electronic polarization and charge transfer. Moreover, they also provide information on the system electronic structure.

A complementary approach to describe the properties of ordered and disordered silica emphasizes its covalent character, representing the system as made of atoms connected by a network of bonds, satisfying fairly restrictive chemical rules. The potential energy is written as the sum of contributions accounting for deviations of current bonding parameters such as bond length, bending, and torsion angles, from standard values measured on a reference structure which represents the model ground state (see, for instance, Ref. 39).

Because of their simplicity and computational efficiency, classical force field models of this type provided an early approach to compute elastic and vibrational properties of covalently bonded network materials and have been extensively used even before the massive development of modern computers. The dependence of these models on a fixed bonding topology, however, represented a major limitation in their application, preventing their usage in atomistic simulations of high-temperature systems.

The limitations of force fields have been greatly eased by the rules introduced by Wooten, Winer, and Weaire (WWW) to interchange bonds ${ }^{40}$ that effectively break the quasistatic picture of traditional force fields. The investigation of lowtemperature amorphous system provided the primary motivation for the development of the WWW algorithm and still represents the great majority of its applications. Amorphous $\mathrm{Si}, \mathrm{Ge}$, or $\mathrm{SiO}_{2}$ samples obtained by a large number of bond interchanges display a concentration of defects in far better agreement with experimental data than obtained by quenching liquid samples whose potential energy is represented by few-body potentials. ${ }^{36,41}$ The radial distribution functions and angle probability distributions ${ }^{42}$ obtained by this method for low-temperature amorphous samples are in good agreement with experimental data. ${ }^{43,44}$

Until recently, models of this type have been used within a static framework or in approaches combining bond switches with energy quenches. ${ }^{45,46}$ We applied a similar force field approach supplemented by bond interchanges within an equilibrium scheme to investigate thermal properties of bulk silica. ${ }^{47}$ The results show that, somewhat surprisingly, the method is able to provide a qualitatively correct view of melting and of the glass transition. In the present study, we use the same method to study structural properties of amorphous silica surfaces. Once again, the simplicity and computational efficiency of the model allow the extensive simulation of large samples at a modest computational cost. This, in turn, brings within the reach of our analysis properties such as roughness that require large samples and good statistics to be determined.

\section{MODEL AND THE SIMULATION METHOD}

The model we use is based on the well-known Keating potential, ${ }^{39}$ expressing the system potential energy as 


$$
E=\sum_{\{i j\}} f_{i j}\left(\left|\mathbf{R}_{\mathbf{i}}-\mathbf{R}_{\mathbf{j}}\right|\right)+\sum_{\{i j k\}} g_{i j k}\left(\theta_{i j k}\right),
$$

where $\left\{\mathbf{R}_{\mathbf{i}}, i=1, \ldots, N\right\}$ are the atoms' positions, $\{i j\}$ indicates the list of atom pairs connected by a covalent bond, and $\theta_{i j k}$ is the angle centered on atom $j$ and connecting atoms $i, j$, and $k$. The $\{i j\}$ list is primarily determined by the system chemistry and, in the most basic formulation of the model, it does not change during simulations. The set of triplets $\{i j k\}$ and, when needed, the corresponding list of quadruplets are derived from the $\{i j\}$ list.

The simplest choice for $f(R)$ is a harmonic term

$$
f_{i j}\left(\left|\mathbf{R}_{\mathbf{i}}-\mathbf{R}_{\mathbf{j}}\right|\right)=\frac{k_{i j}}{2}\left[\left|\mathbf{R}_{\mathbf{i}}-\mathbf{R}_{\mathbf{j}}\right|-R_{i j}^{(0)}\right]^{2},
$$

where $R_{i j}^{(0)}$ is a reference distance for atoms whose atom type matches that of $i j$, and $k_{i j}$ is the corresponding force constant. A similar choice is often made for $g(\theta)$, assumed to be of the form $g_{i j k}\left(\theta_{i j k}\right)=h_{i j k}\left[\cos \theta_{i j k}-\cos \theta_{i j k}^{(0)}\right]^{2} / 2$. In this last expression, $\theta_{i j k}^{(0)}$ is a reference angle for triplets whose type corresponds to that of $i j k$. In the case of covalent solids, $R_{i j}^{(0)}$ and $\theta_{i j k}^{(0)}$ are obtained from a reference crystal structure that plays the role of the ground-state geometry for the model and may differ from the actual (experimental) ground-state structure of the material. Even though the $\theta_{i j k}$ dependence of the energy is not really quadratic in the atomic coordinates, we shall refer to the potential defined by Eq. (1) together with the simplest choice for $f$ and $g$ as the harmonic model. Different choices for $f$ and $g$ can be made and a torsion term could be added to improve the realism of the model with respect to any given material. Moreover, a short-range potential is often added to prevent atoms from occupying nearly the same position, eventually leading to a volume collapse whenever bond interchanges are introduced.

In the case of pure silica, atoms belong to two different species. The covalent and ionic bondings of silica are both succinctly represented by nearest-neighbor bonds, an approximation that is expected to provide only a qualitative description of $\mathrm{SiO}_{2}$. In our computations, the model reference structure is $\beta$-cristobalite $(F d \overline{3} m)$, which is the stable phase of $\mathrm{SiO}_{2}$ at $T>1743 \mathrm{~K}$ and melts into liquid $\mathrm{SiO}_{2}$ at $T=2000 \mathrm{~K}^{48}$ To reproduce the lattice constants and the interatomic distances in the reference structure, we set $R_{\mathrm{SiO}}^{(0)}$

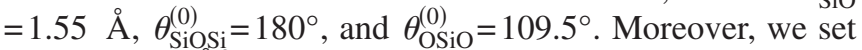
$k_{\mathrm{SiO}}=10 \mathrm{eV} / \AA^{2}, h_{\mathrm{SiOSi}}=0.75 \mathrm{eV}$, and $h_{\mathrm{OSiO}}=4.32 \mathrm{eV}$. The bending constants are the same as those used in previous studies of silica based on the Keating potential..$^{35,49,50}$ The $\mathrm{Si}-\mathrm{O}$ stretching constant, instead, is approximately half of the value used in previous works for quartz. The primary reason for weaker $k_{\mathrm{SiO}}$ is related to our assumption of the ideal $\mathrm{Fd} \overline{3} m$ structure, having straight $\mathrm{Si}-\mathrm{O}-\mathrm{Si}$ angles, as the reference structure for our $\beta$-cristobalite model. The most likely experimental structure, instead, belongs to space group $I \overline{4} 2 d$, having slightly bent $\mathrm{Si}-\mathrm{O}-\mathrm{Si}$ angles. ${ }^{51,52}$ This difference implies that the stretching constants of our model account for energy changes that in reality are due to a combination of bending, stretching, and torsion, resulting into effective force constants weaker than those describing the pure stretching of
TABLE I. Comparison of the elastic constant computed by our model at $T=0 \mathrm{~K}$ with the experimental values for amorphous silica at ambient conditions (Ref. 54) and with the computational data for $\beta$-cristobalite computed by the potential of Ref. 27 at high temperature ( $T=1800 \mathrm{~K})($ Ref. 53).

\begin{tabular}{lcccc}
\hline \hline Sample & $\begin{array}{c}\mathrm{B} \\
(\mathrm{GPa})\end{array}$ & $\begin{array}{c}\mathrm{C}_{11} \\
(\mathrm{GPa})\end{array}$ & $\begin{array}{c}\mathrm{C}_{12} \\
(\mathrm{GPa})\end{array}$ & $\begin{array}{c}\mathrm{C}_{44} \\
(\mathrm{GPa})\end{array}$ \\
\hline Our model $\beta$-cristobalite $^{\text {a }}$ & 37 & 60 & 26 & 27 \\
Amorphous Exp. $^{\mathrm{a}}$ & 37 & 51 & & 20 \\
Amorphous Sim. $^{\text {b }}$ & & 42.0 & 5.2 & 40.8 \\
\hline \hline
\end{tabular}

${ }^{\text {aReference }} 54$.

${ }^{\mathrm{b}}$ Reference 53 .

Si-O bonds. A direct comparison of bulk modulus and elastic constants with those of $\beta$-cristobalite is indeed difficult because the structure is stable at high temperature and only computational estimates are available for its elastic constants..$^{53}$ The values computed at $T=0$ by our model and listed in Table I are comparable to those measured in amorphous silica at ambient conditions ${ }^{54,55}$ or in other phases of silica which are stable at low temperature ${ }^{56}$ Despite this very qualitative validation, it is clear that a description of elastic and vibrational properties of even semiquantitative quality requires a more sophisticated model, including at least torsion contributions, and perhaps longer range interactions.

In our model, the function $f$ retains the simple harmonic form in the vicinity of $R_{i j}^{(0)}$, but deviates at longer separations according to

$$
f(R)=\left\{\begin{array}{cc}
\frac{1}{2} k\left[R-R^{(0)}\right]^{2}, & R-R^{(0)} \leq \delta \\
\frac{1}{2} k\left[\delta^{2}+0.2\left(\left|R-R^{(0)}\right|-\delta\right)^{2}\right], & R-R^{(0)}>\delta,
\end{array}\right.
$$

where $\delta=0.3 \AA$.

The angular term is modified in such a way to decrease its strength when the product of the bending distances is larger than a preset value

$$
\begin{aligned}
& \frac{2 g_{i j k}(\theta)}{\left[\cos \theta_{i j k}-\cos \theta_{i j k}^{0}\right]^{2}} \\
& \quad=\left\{\begin{array}{cc}
h_{i j k}, & R_{i j} R_{j k} \leq T_{i j k} \\
h_{i j k} \exp \left[-\left(R_{i j} R_{j k}-T_{i j k}\right)\right], & R_{i j} R_{j k} \geq T_{i j k},
\end{array}\right.
\end{aligned}
$$

where $T_{i j k}=\left(R_{i j}^{(0)}+\delta\right)\left(R_{j k}^{(0)}+\delta\right)$. The short-range potential is of the form

$$
U=\sum_{i \neq j}\left(R_{c}-R_{i j}\right)^{4}, \quad R_{i j} \leq R_{c},
$$

where $R_{c}=2.4 \AA$ for like particles and $R_{c}=1.5 \AA$ for unlike particles. This choice is similar to the one made in Ref. 42 .

Although simple, these modifications have important consequences. First of all, the original harmonic potential greatly exaggerates the tendency to a negative expansion coefficient often observed in tetrahedral network materials. In 
(a)
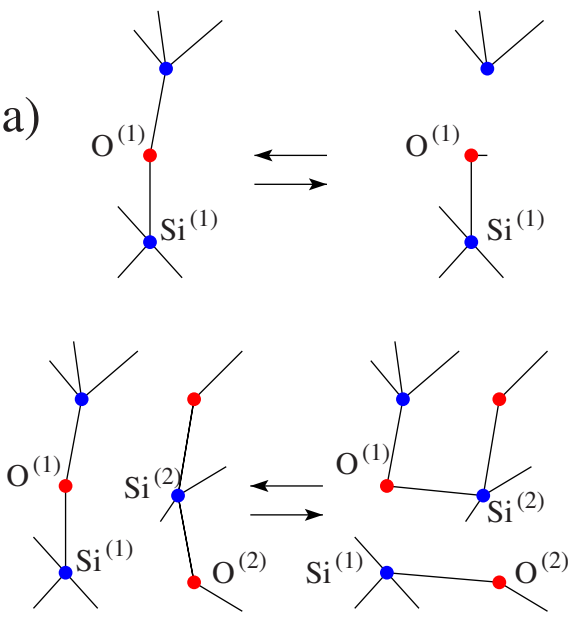

(b)

FIG. 1. (Color online) Schematic drawings of the atomistic mechanisms to (a) break and form bonds and (b) interchange bonds.

the case of $\beta$-cristobalite, for instance, experimental measurements covering the $573 \mathrm{~K} \leq T \leq 2000 \mathrm{~K}$ interval show that an initial volume expansion with increasing $T$ up to $\sim 1300 \mathrm{~K}$ combines with a slight contraction for $1300 \leq T$ $\leq 2000 \mathrm{~K}$ to give rise to a nearly constant volume over the entire temperature range. ${ }^{48}$ Simulations performed with the harmonic potential, instead, show a volume contraction of more than $5 \%$ over the same $T$ range. The anharmonicity introduced by the modification of Eqs. (3) and (5) reduces the volume contraction for $T<1000$ and gives rise to slightly positive expansion coefficient for $T>1000 \mathrm{~K}$. The compensation of these opposite trends results into an equilibrium volume at $T=2000 \mathrm{~K}$ that is nearly the same of the $T$ $=0 \mathrm{~K}$ volume, thus qualitatively (but not quantitatively) reproducing the experimental picture. Moreover, both modifications of Eqs. (3) and (4) are meant to mimic the drastic weakening of covalent bonds whenever the corresponding bond length increases by more than $\sim 10 \%$.

The major modifications with respect to the original Keating potential, however, are represented by rules to break, form, or interchange bonds. Breaking a bond requires a large energy $E_{b}=4.8 \mathrm{eV}$ and releases the stretching and bending energies associated to the removed bond. Forming a bond releases an equally large $E_{b}$ energy, but the new bond is born with stretching and bending energy contributions that might be not negligible. Bond interchanges are attempted by first selecting at random a $\mathrm{SiO}$ bond, say $\mathrm{Si}^{(1)}-\mathrm{O}^{(1)}$. Then, a second oxygen $\left[\mathrm{O}^{(2)}\right]$ is chosen at random within a short distance from $\mathrm{O}^{(1)}$ and, again at random, one of its Si neighbors $\left(\mathrm{Si}^{(2)}\right)$ is selected. As a last step, we discontinuously replace the $\mathrm{Si}^{(1)}-\mathrm{O}^{(1)}$ and $\mathrm{Si}^{(2)}-\mathrm{O}^{(2)}$ bonds with $\mathrm{Si}^{(1)}-\mathrm{O}^{(2)}$ and $\mathrm{Si}^{(2)}-\mathrm{O}^{(1)}$ bonds (see Fig. 1 ). The variation in the bonding topology is accepted or rejected by the Monte Carlo (MC) procedure described below. The mechanism is virtually identical to the WWW one, ${ }^{40,57}$ the only (minor) difference being that we do not impose that $\mathrm{Si}^{(1)}$ and $\mathrm{Si}^{(2)}$ to be covalently bonded to the same $\mathrm{O}$ atom, even though in practice this is invariably the case up to fairly high $T$. We also emphasize that the computational approach is practically the same as the one introduced in Ref. 57. In this respect, the major difference is that the authors of Ref. 57 couple bond interchanges with a strict local energy minimization to investigate lowtemperature materials, while we aim at high-temperature properties and we resort to an equilibrium simulation method with respect to either particles and bonds.

In other terms, the system represented by our model consists of atoms and bonds. These last are coded into discrete variables that tell which atom pairs are linked by a covalent bond. The bonding state of atoms $i$ and $j$ is an independent variable, not explicitly determined by their separation, even though the probability of long bonds is vanishingly small since their energy increases quadratically with increasing length.

In what follows, we are primarily concerned with the simulation of finite slabs, periodically repeated in two dimensions to represent extended planar surfaces. Monte Carlo is used to sample the phase space of the system, combining single atom moves with variations in the network of bonds. The short range of the potential and the slow variation of the bonding configuration ensure an almost ideally linear scaling of the computational time with system size. Strictly speaking, the short-range repulsive potential requires an effort proportional to the square of the number of atoms, but its shortrange and simple analytical form, together with a standard list of neighbors, reduces the cost of this term to a small fraction of the total CPU time up to the sizes considered in the present study. An efficient implementation of all parts of our program allows us to perform $2 \times 10^{8}$ attempted atom moves and an equal number of bond interchange attempts per day on a single OPTERON CPU. In what follows, MC steps indicate the combination of one attempted single-atom displacement and one attempted bond breaking, formation, and interchange, performed in sequence. We verified that selecting at random the type of moves to attempt gives results that are indistinguishable from those of the regular sequence that we adopted. The slight violation of microscopic reversibility involved in our choice is in practice concealed by the low-acceptance ratio for the changes of the bond topology that makes immaterial the precise composition of short move sequences. In part of our simulations, changes of the sample cross section are attempted every $1000 \mathrm{MC}$ steps in order to enforce the condition of zero in-plane stress. Even at this slow rate, attempted changes in the surface area, which involve the recomputation of the system energy from scratch, represent a sizable fraction of the total CPU time.

\section{Summary of results for bulk $\mathrm{SiO}_{2}$}

The model for $\mathrm{SiO}_{2}$ described above has been used, first of all, to simulate homogeneous phases of silica. ${ }^{47}$ The results support the validity of the model and give us confidence in the description of the surface properties described in Sec. III. Computations have been carried out by MC in the NPT ensemble using a procedure closely related to the one described in Sec. II. The results, reported in Ref. 47, show that the model is able to describe the melting of silica, at a temperature estimated at $T_{m}=2200 \mathrm{~K}$ (see Fig. 2), to be com- 


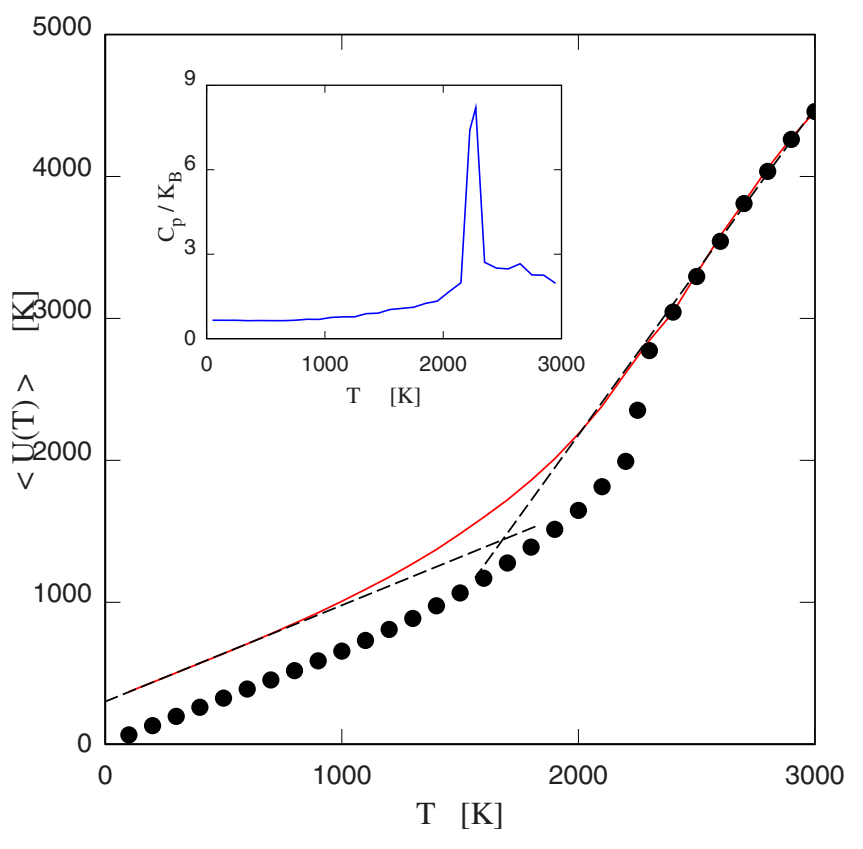

FIG. 2. (Color online) Average potential energy per atom of bulk silica as a function of temperature. Solid dots: heating stage. Full line: cooling stage. Dashed lines: linear interpolation of the low- and high- $T$ portions of $U(T)$, crossing at $T_{g}=1680 \mathrm{~K}$. (Inset) Constant pressure specific heat per atom on heating.

pared to the experimental melting temperature $T_{M}=2000 \mathrm{~K}$ for $\beta$-cristobalite. The average potential energy computed during the slow annealing of the silica sample down to room temperature, also shown in Fig. 2, displays two linear ranges of different slope, matching at $T=1680 \pm 50 \mathrm{~K}$. In atomistic simulations, this behavior is usually interpreted in terms of a glass transition, thus estimated at $T_{g}=1680 \pm 50$. The simulation result can be compared to a measured $T_{g}=1480 \mathrm{~K}$ for slow cooling and $T_{g} \sim 1600 \mathrm{~K}$ for fast quenches. ${ }^{58}$ At all temperatures and for all phases, almost every $\mathrm{Si}$ atom is fourfold coordinated and almost every $\mathrm{O}$ atom is twofold coordinated, in agreement with experimental data. ${ }^{43,44}$

Above $T_{m}$ during heating and above $T_{g}$ during cooling, the mean-square displacement of atoms grows linearly with the number of MC steps, showing that the motion of atoms is unbound, consistently with liquidlike diffusion. Comparison of atomic mobilities computed by MC during cooling to experimental data for the diffusion coefficient in disordered silica phases allows us to establish a rough correspondence with a real time scale. The diffusion coefficient of $\mathrm{Si}$ atom has been measured as a function of temperature and an interpolation of the experimental data is provided in Ref. 59. Using this expression slightly beyond its definition range (thus performing an extrapolation), we obtain a diffusion coefficient $D=5.2 \times 10^{-15} \mathrm{~cm}^{2} / \mathrm{s}$ at $T=1800 \mathrm{~K}$ and $D=2.5$ $\times 10^{-13} \mathrm{~cm}^{2} / \mathrm{s}$ at $T=2000 \mathrm{~K}$. On the other hand, from the linear part of the mean-square displacement as a function of MC steps, we obtain $D=6.3 \times 10^{-5} \AA^{2} / 10^{6}$ MC steps at $T$ $=1800 \mathrm{~K}$ and $D=3.0 \times 10^{-3} \AA^{2} / 10^{6} \mathrm{MC}$ steps at $T$ $=2000 \mathrm{~K}$. All the quoted simulation results refer to the cooling stage from the liquid. A simple conversion allows us to estimate that our standard run of $10^{8} \mathrm{MC}$ steps corresponds

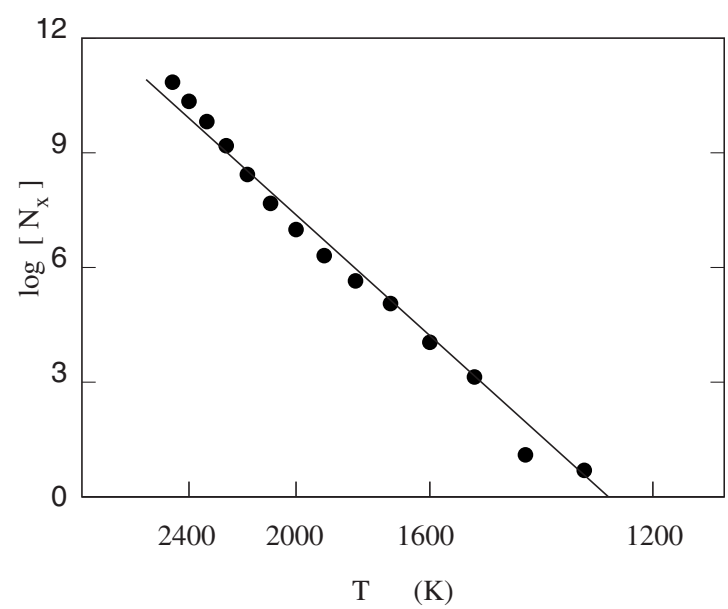

FIG. 3. Arrhenius plot for the average number $N_{x}$ of bond interchanges observed during a MC run of $10^{8}$ steps. Dots: simulation results; full line: linear interpolation, whose slope is $-2.2 \mathrm{eV}$.

to $0.13 \mathrm{~ms}$ at $T=1800 \mathrm{~K}$ and to $0.12 \mathrm{~ms}$ at $T=2000 \mathrm{~K}$. This estimate is purely qualitative and the result would differ, even substantially, if we consider any other activated process. Nevertheless, this simple analysis suggests that MC with bond interchanges greatly expands the time scale covered by simulation.

Atomic mobility and diffusion are, of course, strictly related to bond interchanges, whose probability is different from zero only above the glass temperature $T_{g}$ of our model. An Arrhenius plot of the probability of bond interchanges is shown in Fig. 3. A linear fit of this curve provides an estimate for the activation energy of bond interchanges of $\Delta$ $=2.2 \mathrm{eV}$, in fair agreement with the experimental data $(\Delta$ $=2.9 \mathrm{eV}$ ) reported in Ref. 60 .

The melting transition highlighted in our simulations has an intriguing similarity with the entropy jump found in Ref. 45, observed, however, at constant low $T$ by a simulation method (the so-called activation-relaxation technique, ART) that combines bond interchanges and quenches. In other terms, the entropy jump of Ref. 45 appears to be a transition driven by an external perturbation (for instance, by irradiation, creating topological defects), while the melting we found in Ref. 47 is an equilibrium phase transition.

The radial distribution functions computed during our simulations are shown in Fig. 4. At ambient temperature, they agree at least qualitatively and, to a large extent, semiquantitatively with experimental data provided by $\mathrm{x}$-ray and neutron diffractions. 43,44

\section{SIMULATION OF THE DRY $\mathrm{SiO}_{2}$ SURFACE}

We consider a $\beta$-cristobalite slab obtained by replicating along the $x y$ plane an orthorhombic simulation cell made by $9 \times 9 \times 6$ conventional unit cells of $8 \mathrm{SiO}_{2}$ molecules, corresponding to 11664 atoms. The simplest procedure to cut the surfaces along unit-cell boundaries while maintaining the sample stoichiometry produces two inequivalent surfaces: one exposing only oxygen atoms and the other exposing only silicon atoms [see Fig. 5(a)]. We restore the up-down sym- 


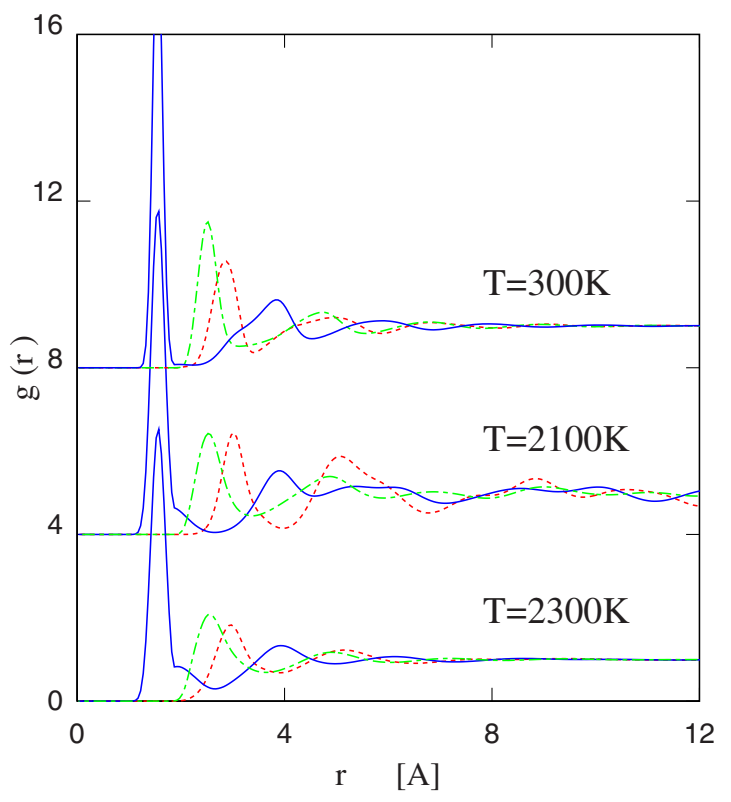

FIG. 4. (Color online) Radial distribution function of the bulk silica in the equilibrium liquid $(T=2300 \mathrm{~K})$, undercooled liquid $(T=2100 \mathrm{~K})$, and amorphous phase $(T=300 \mathrm{~K})$. Full line (blue): $\mathrm{Si}-\mathrm{O}$ correlation; dotted line (red) Si-Si correlation; dashed-dotted line (green): $\mathrm{O}-\mathrm{O}$ correlation. The $T=2100 \mathrm{~K}$ and $T=300 \mathrm{~K}$ plots have been shifted by four units along the vertical direction for the sake of clarity.

metry of the slab by moving one half of the $\mathrm{O}$ atoms from the oxygen-only surface to the Si-only surface. The starting geometry obtained by this simple construction is shown in Fig. 5(b). A visual impression of size and aspect ratios of the simulated samples is provided in Fig. 6.

At the lattice constant $\left(a_{0}=7.166 \AA\right)$ of the bulk crystal phase at $T=0 \mathrm{~K}$, the slab exposes two planar and square surfaces of $4160 \AA^{2}$ cross area each. The surface energy of the as-cut sample is $2995 \mathrm{erg} / \mathrm{cm}^{2}$, or $0.18695 \mathrm{eV} / \mathrm{A}^{2}$, corresponding to two broken bonds per surface unit cell. This surface energy, comparable to that of highly refractory

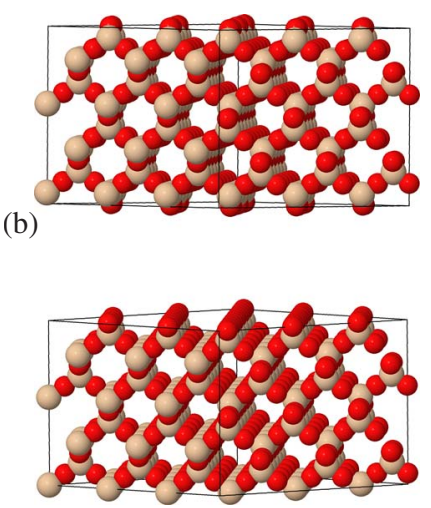

(a)

FIG. 5. (Color online) (a) Asymmetric silica slab obtained by cutting a $\beta$-cristobalite crystal along unit-cell boundaries. (b) Symmetrized version of the slab shown in (a). Brown (light) atoms: Si; red (dark) atoms: O. For the sake of clarity, the figure shows a smaller sample than used in the simulations.

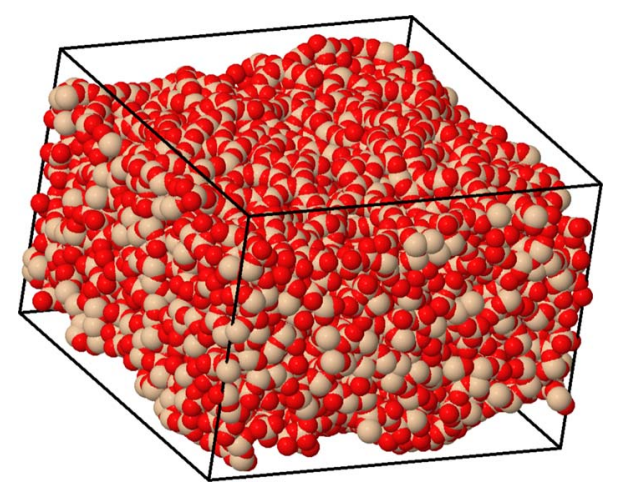

FIG. 6. (Color online) Simulation snapshots of the amorphous slab at $T=300 \mathrm{~K}$. Brown (light) atoms: $\mathrm{Si}$; red (dark) atoms: O.

metals, ${ }^{61}$ is of course the result of the idealized process of cutting the surface along a geometric plane, leaving behind a regular array of dangling bonds.

Here and in what follows, the surface energy $u_{s}$ is defined by comparing the average potential energy of the slab $U_{\text {slab }}$ to that of a homogeneous sample $U_{b u l k}$ with the same number of atoms, computed during the simulations described in Ref. 47 ,

$$
u_{s}(T)=\left[U_{\text {slab }}(T)-U_{\text {bulk }}(T)\right] / 2 A,
$$

where $A$ is the surface area at temperature $T$. The factor of 2 in the denominator accounts for the fact that the slab has two equivalent and nearly planar surfaces. The first part of the simulations described below has been carried out at constant shape and size of the simulation cell and $A$ is a constant. As discussed in some more details below, the cross area of the simulation cell has been treated as a dynamical variable in the last stage of our simulations and in such a case, $A$ is the thermal average of the cross area.

The as-cut slab has been first equilibrated by $\mathrm{MC}$ at $T$ $=2600 \mathrm{~K}$, i.e., well beyond the bulk melting temperature $\left(T_{m}=2200 \mathrm{~K}\right)$ of the model. At this stage, we increased slightly $\left(a_{0}=7.32 \AA\right)$ the lattice constant of the unit cell to match the average density of the homogeneous liquid at $T$ $=2600 \mathrm{~K}$. Despite the fairly high simulation temperature, the number of dangling bonds decreases steadily and rapidly during the equilibration stage, greatly reducing the surface energy. At $T=2600 \mathrm{~K}$ the initial crystal-like sample quickly reverts to the liquid state. After a few runs of $10^{8} \mathrm{MC}$ steps, the surface energy at $T=2600 \mathrm{~K}$, computed by comparisons to the energy of a $\mathrm{SiO}_{2}$ sample of the same size and at the same temperature, turns out to be $u_{s} \sim 200 \mathrm{erg} / \mathrm{cm}^{2}$, already providing the scale of the thermodynamic surface properties expected for our slab. The average number of broken bonds in the entire system is about 10. Even this low value is 1 order of magnitude larger than the average number of broken bonds in the homogeneous liquid phase (about 2 for the same number of atoms) and it might be interpreted as a surface effect or as an indication that the late stages of equilibration for the bond network, requiring the long-range migration of defects, might take much longer than the total duration of our simulations. In any case, both the concentration and the effect of dangling bonds on the surface properties are negli- 
gible, in qualitative agreement with experimental data and with previous computational results. ${ }^{17}$

The liquid slab has been progressively cooled down to $T=0 \mathrm{~K}$ in regular steps of $100 \mathrm{~K}$. At this stage, the choice of the simulation ensemble plays a crucial role. On one hand, above the melting temperature, a MC procedure that allows changes of the simulation cell would tend to shrink the surface of liquid samples driven by surface tension. This, in turn, would alter the initial $9 \times 9 \times 6$ aspect ratio that we selected in order to achieve a sufficient thickness together with a large surface area. On the other hand, a simulated annealing carried out at constant periodicity would be unable to release the stress in the $x y$ plane below the glass transition temperature. As a practical compromise, we decided to perform a first annealing down to $T=0 \mathrm{~K}$ at constant simulation cell, accepting the fact that the low-temperature samples are strained. Each of the partially equilibrated samples is further relaxed during a second stage of the annealing, which includes moves that change the surface area $(N \Sigma T$ ensemble, at vanishing in-plane stress), thus effectively removing the $x y$ strain. At each temperature, the first annealing (at constant area) consists of $3 \times 10^{8} \mathrm{MC}$ steps, the last third being considered for statistics. The second stage of relaxation (at variable area) consists of $2 \times 10^{8} \mathrm{MC}$ steps, half of which are considered for computing equilibrium properties. As expected, the second relaxation stage has the effect of decreasing slightly the surface area for the liquid samples. However, since all samples to be simulated at $N \Sigma T$ conditions have already been generated and partially equilibrated during the quench at NVT conditions, changes in the cross area that might take place at high $T$ are not propagated all the way down to $T=0 \mathrm{~K}$. In any case, even at the highest temperature considered in our simulations, the variation of the inplane periodicity is relatively minor and thus all the samples are, to a large extent, geometrically similar.

The simulation data for the average potential energy $\left[U_{\text {slab }}(T)\right]$ of the slab as a function of temperature are qualitatively indistinguishable from those for the bulk phase displayed in Fig. 2. The glass temperature of the slab has been determined using the same method briefly described in Sec. II, i.e., by crossing the linear interpolations to $U_{\text {slab }}(T)$ at low $T$ and at high $T$. The result provides an estimate of the glass temperature for the slab $\left(T_{g}=1660 \pm 50 \mathrm{~K}\right)$ that, within an estimated error bar of $50 \mathrm{~K}$, cannot be distinguished from the bulk value $\left(T_{g}=1680 \pm 50 \mathrm{~K}\right)$. This result already suggests that the thickness of the slab is sufficient to guarantee bulklike behavior to the sample.

The essential role of releasing the in-plane stress when computing thermodynamic surface properties is emphasized in Fig. 7, comparing the computational estimate of the surface energy at fixed size of the simulation cell, and upon allowing the simulation box to change its cross section. Strictly speaking, the two sets of data should coincide at $T$ $>T_{g}$, since atomic diffusion already provides a mechanism to relax the in-plane strain. Therefore, differences in the two sets of data at high temperature highlight the difficulty of enforcing the condition of zero-average stress by atomic diffusion alone. This difficulty is apparently related to the viscosity of silica that remains high even at temperatures beyond the melting point. On the other hand, allowing

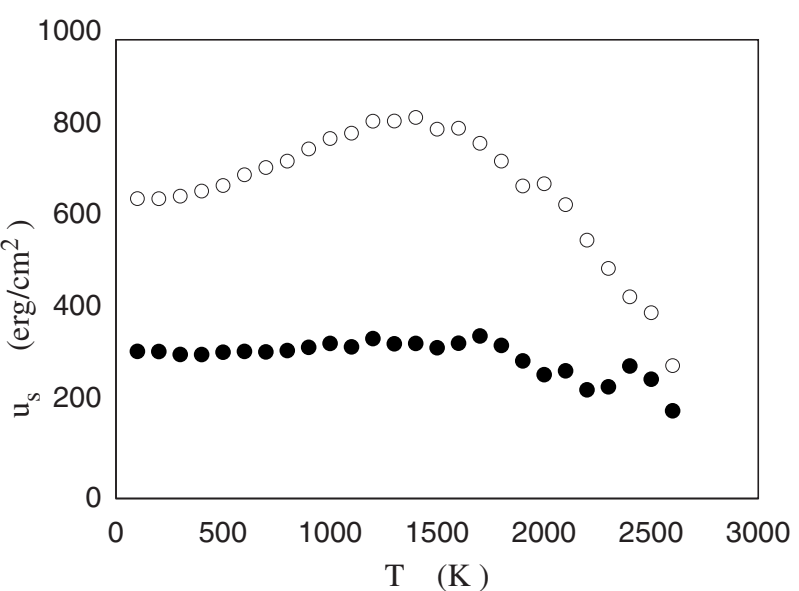

FIG. 7. Temperature dependence of the surface energy. Full dots: simulation results upon releasing the in-plane stress (see text). Open dots: simulation results before releasing the in-plane stress, i.e., obtained at constant in-plane periodicity.

fluctuations of the surface area quickly removes the average in-plane stress. This has been verified by performing additional $N \Sigma T$-MC runs at selected (low) temperatures. The average value of the cross area as well as the estimate for the surface energy did not change significantly after the first $10^{8}$ MC steps of $N \Sigma T$ equilibration.

The data allow us to compute the surface entropy by thermodynamic integration, according to the relation

$$
\Delta S_{s}=S[s l a b]-S[b u l k]=\int_{T_{0}}^{T} \frac{\Delta C_{p}\left(T^{\prime}\right)}{T^{\prime}} d T^{\prime},
$$

where $\Delta C_{p}(T)=\left[C_{p}(\right.$ slab $)-C_{p}($ bulk $\left.)\right](T)$ and $T_{0}$ is a suitable reference temperature. The surface entropy is then defined as

$$
s_{s}(T)=\Delta S_{s}(T) / 2 A
$$

and the surface tension

$$
\gamma(T)=u_{s}(T)-T s_{s}(T) .
$$

Moreover, neglecting the (fairly small) temperature dependence of the surface area, we can write

$$
\begin{aligned}
s_{S}(T) & =\Delta S_{s}(T) / 2 A \sim \int_{T_{0}}^{T} \frac{1}{T^{\prime}} \frac{\Delta C_{p}\left(T^{\prime}\right)}{2 A} d T^{\prime} \\
& =\int_{T_{0}}^{T} \frac{1}{T^{\prime}} \frac{d}{d T^{\prime}} u_{s}\left(T^{\prime}\right) d T^{\prime} .
\end{aligned}
$$

To simplify the integration task, we first fit the surface energy $u_{s}(T)$ by a simple polynomial

$$
u_{s}(T)=u_{0}+b T^{2}+c T^{3},
$$

where the linear term is absent because both the energy of the slab and the energy of the bulk will show the same temperature dependence in the limit of low temperatures and harmonic dynamics

$$
U_{s l a b}=U_{s l a b}^{0}+\frac{3}{2} N K_{B} T+o\left(T^{2}\right),
$$




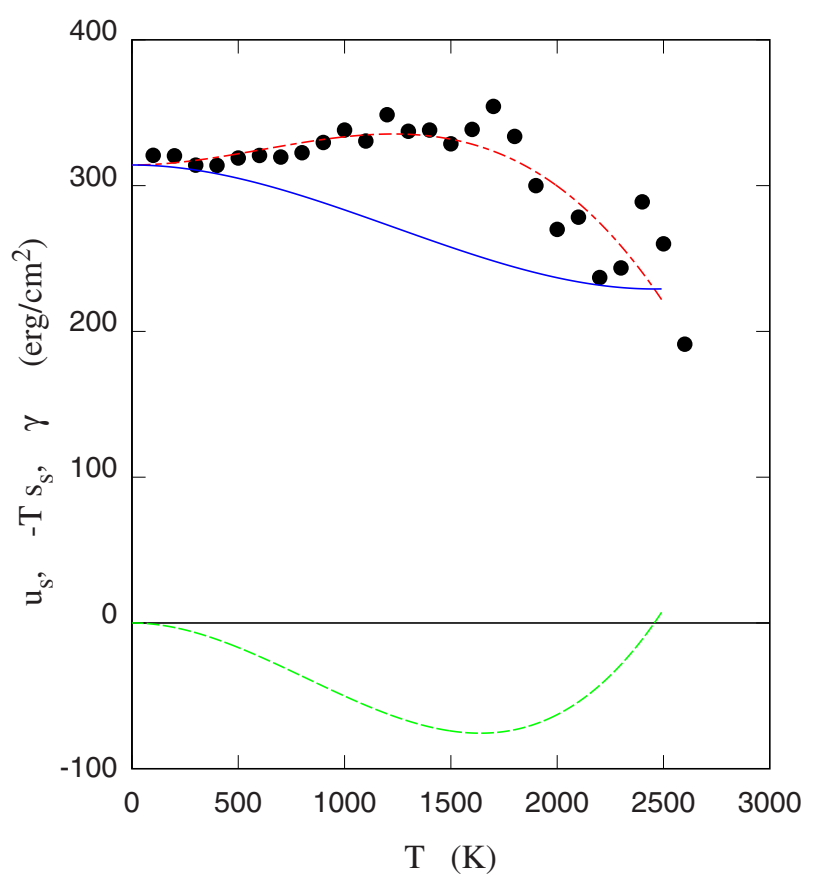

FIG. 8. (Color online) Thermodynamic surface properties from MC simulations upon releasing the in-plane stress. Solid dots: simulation results for the surface energy $u_{s}$. Dashed-dotted line (red): polynomial fit to the simulation data (see text). Dashed line (green): entropy contribution $-T s_{s}$ to the surface tension. Full line (blue): surface tension $\gamma$.

$$
U_{\text {bulk }}=U_{\text {bulk }}^{0}+\frac{3}{2} N K_{B} T+o^{\prime}\left(T^{2}\right),
$$

where $N$ is the (same) number of atoms in the slab and in the bulk. The two linear terms, therefore, cancel each other in the difference defining the surface energy. In this way, we obtain

$$
\begin{aligned}
s_{S}(T) & =\int_{T_{0}}^{T}\left[2 b+3 c T^{\prime}\right] d T^{\prime} \\
& =\left[2 b T+\frac{3}{2} c T^{2}\right]-\left[2 b T_{0}+\frac{3}{2} c T_{0}^{2}\right] .
\end{aligned}
$$

In what follows, we set $T_{0}=0$ and compute a quantity that looks like an absolute surface entropy for the slab. The $\chi^{2}$ fit of the surface energy gives $b=9.111 \times 10^{-5} \mathrm{erg} / \mathrm{cm}^{2} \mathrm{~K}^{2}$ and $c=-4.156 \times 10^{-8} \mathrm{erg} / \mathrm{cm}^{2} \mathrm{~K}^{3}$. The result for the surface entropy is shown in Fig. 8.

The combination of $-T s_{s}$ with $u_{s}$ allows us to estimate the surface free energy, whose value per unit area is the surface tension $\gamma$. The result of this last elaboration of the simulation data is shown again in Fig. 8. It is apparent that the potential energy part represent the major contribution to the surface tension up to fairly high temperature $(T>1000 \mathrm{~K})$. However, up to $T \sim 2000 \mathrm{~K}, u_{s}$ is nearly constant and the entropy contribution is responsible for most of the variations of $\gamma$ with $T$. Remarkably, the value of the computed surface tension agrees well with experimental estimates $(\gamma$ $\sim 300 \mathrm{erg} / \mathrm{cm}^{2}$, see Ref. 11). Because of the large uncertain-

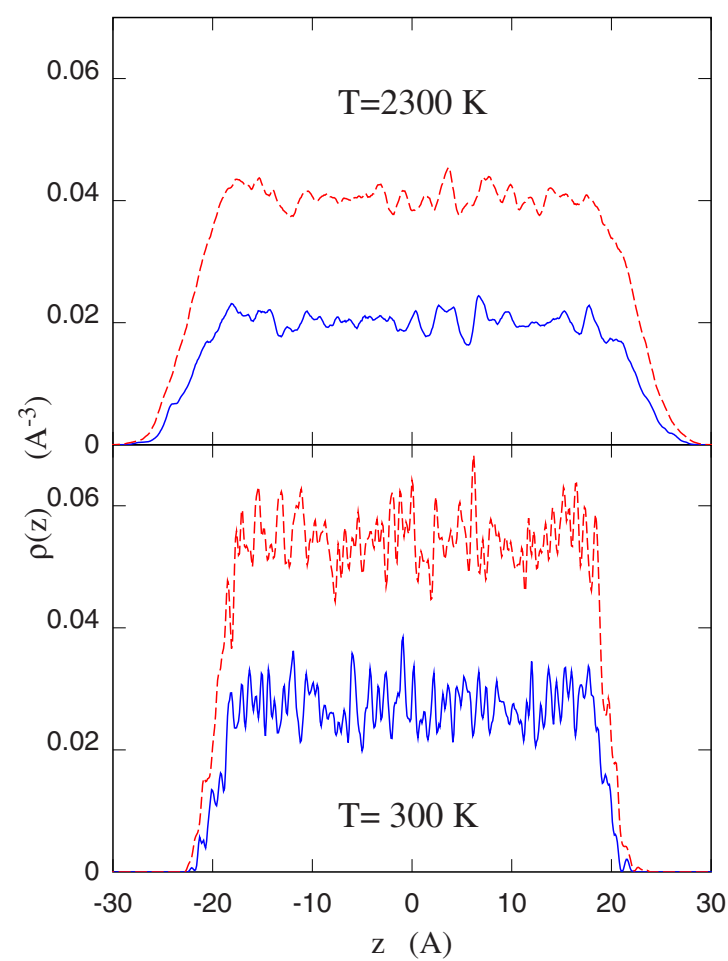

FIG. 9. (Color online) Atomic density profile along the normal to the surface at two different temperatures in the liquid phase ( $T$ $=2300 \mathrm{~K})$ and in the low-temperature $(T=300 \mathrm{~K})$ amorphous phase. Full (blue) line: silicon atoms; dashed (red) line: oxygen atoms.

ties in the experimental measurements, this good agreement should not be overemphasized. Nevertheless, our computational results might indeed represent one of the first successful computations of the surface tension of amorphous silica over a wide temperature range. The thermodynamic results discussed above are complemented by the determination of a wide range of structural and pseudodynamical properties, these last representing dynamical properties as a function of the number of MC steps.

The density profile for silicon and oxygen atoms along the direction $z$ perpendicular to the surface is shown in Fig. 9. This figure confirms that the simulated slab has a fairly wide bulklike region of nearly constant density and thus its width is adequate for our purposes. Comparison of the profiles at high $(T=2300 \mathrm{~K})$ and low $(T=300 \mathrm{~K})$ temperatures gives a visual impression of the thermal expansion of the slab. The width of the surface region is sometimes measured by the separation $\Delta_{10}^{90}$ of the two parallel planes at which the density is $10 \%$ and $90 \%$ of the bulk value. At low $T, \Delta_{10}^{90}$ turns out to be $\sim 3 \AA$, growing to $\Delta_{10}^{90}=5 \AA$ in the liquid phase at $T$ $=2300 \mathrm{~K}$. At all temperatures, oxygen is the atom type most likely to be first encountered in moving toward the surface. At high temperature $(T \geq 1000 \mathrm{~K})$, this reflects almost exclusively the 1:2 stoichiometry of $\mathrm{SiO}_{2}$ and also the significantly larger radius of $\mathrm{O}^{=}$with respect to that of $\mathrm{Si}^{4+}$. In fact, rescaling the $\mathrm{Si}$ profile by a constant factor of 2 brings it into nearly perfect superposition with the oxygen profile. At low temperature, however, a slight but statistically significant relative enhancement of the oxygen concentration is ob- 
served in a narrow $(\sim 1 \AA)$ outermost surface layer. These observations are consistent with experimental evidence provided by $\mathrm{x}$-ray photoemission spectroscopy ${ }^{7}$ measurements carried out at room temperature. Analysis of the bonding topology resulting from the simulation shows that at all temperatures almost every atom satisfies its ideal coordination (fourfold for silicon and twofold for oxygen). In other words, there is no significant enhancement of dangling bonds at the surface. Also in this case, the simulation results agree at least qualitatively with experimental data obtained by electron spin resonance (ESR) (Ref. 8) and by XPS. ${ }^{9}$

Previous simulation studies of amorphous silica surfaces revealed a sizable concentration of twofold rings, consisting of short $\mathrm{Si}-\mathrm{O}-\mathrm{Si}-\mathrm{O}-\mathrm{Si}$ sequences closing on themselves. In our simulations, we observe instead a fairly low concentration of these topological defects, numbering, on average, less than 40 at $T=300 \mathrm{~K}$ and about 70 at $T=2000$ for our sample of $3888 \mathrm{SiO}_{2}$ formula units. Their density, however, is higher at the edge than well inside the slab, confirming their surface origin. Threefold rings, representing the immediate generalization of the twofold rings definition, are far more abundant and their distribution is already more uniform over the entire slab than in the twofold rings case.

At $T>T_{g}$, our simulations are able to detect atomic diffusion, whose general aspects are very similar to those found in the bulk case. As expected, the mobility of surface atoms is higher than that of atoms located deeper in the slab, as we verified by computing the mean-square displacement of the 200 outermost atoms on each side of the slab. Moreover, again as expected, the mean-square displacement of surface atoms is very anisotropic, being high along the surface plane and practically vanishing along the $z$ direction. The relative advantage of surface over bulklike atoms depends on temperature, increasing with decreasing temperature, thus pointing to a lower activation energy for diffusion at the surface. Unfortunately, diffusion decreases too rapidly with decreasing temperature to be able to extract a reliable estimate for the bulk and surface barrier to diffusion from an Arrhenius plot. Longer runs, however, could be able to provide such an estimate.

One of the most important parameters characterizing the structure of liquid and solid surfaces is the so-called roughness. In our simulations, roughness has been characterized by computing the height-height correlation function over a wide temperature range. First of all, we define the height $h(x, y)$ at position $(x, y)$ along the surface by moving a spherical probe from outside toward the surface. The height $h(x, y)$ is defined as the $z$ coordinate of first contact with atoms on the surface, where contact is defined by crossing the minimum separation of $3 \AA$ for oxygen and $2.5 \AA$ for silicon. The data computed on a $100 \times 100$ grid on the simulated surface have been used to determine the radial average of $\langle h(0) h(r)\rangle$. The results confirm the expected roughness of the liquid surface and provide a direct view of the progressive smoothening in crossing the glass point moving toward the amorphous phase (see Fig. 10). These results, pointing to enhanced atomic mobility and shorter relaxation times at the surface, agree with recent theoretical results ${ }^{62}$ and other simulation data ${ }^{63,64}$ and contradict a theoretical prediction of super-roughness in amorphous surfaces. ${ }^{65}$ The accuracy of

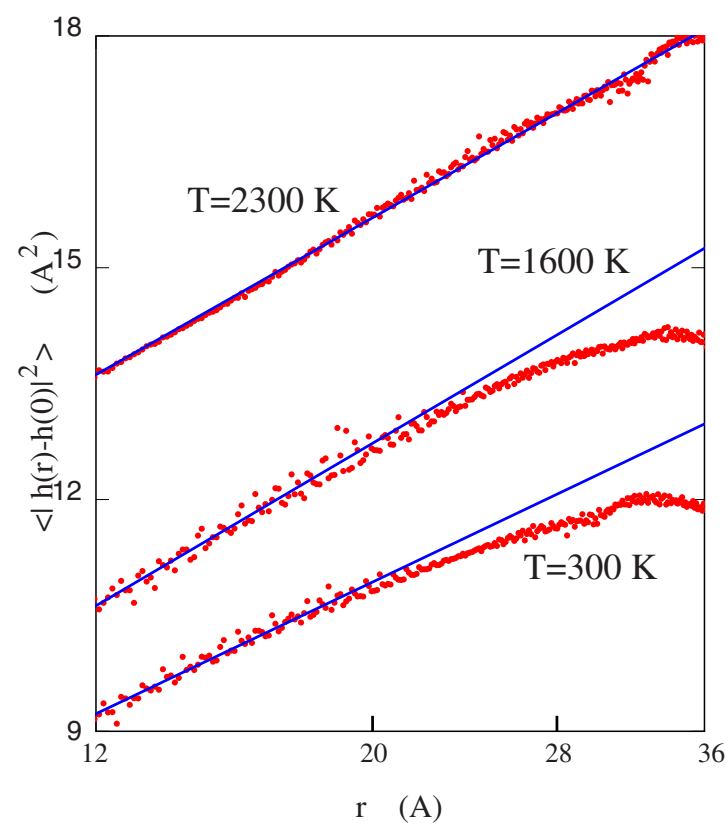

FIG. 10. (Color online) Height-height correlation function as a function of in-plane distance at three different temperatures. Straight lines represent a logarithmic interpolation over the $12 \leq r$ $\leq 20 \AA$ range.

our simulations, limited mainly by the relatively short distances spanned by our slab, does not allow us to determine the roughening transition temperature that cannot be distinguished from the glass point.

Although roughness and smoothing can be defined only in terms of the $\langle h(r) h(0)\rangle$ shown in Fig. 10, the trend toward smoothing below $T_{g}$ can also be seen (although not easily and certainly not quantitatively) also from topography maps of the silica surface, shown in Fig. 11.

\section{SUMMARY AND CONCLUSIONS}

A force field model of the Keating type, supplemented by rules to break, form, and interchange covalent bonds, has been applied to simulate the dry amorphous silicon surface. Computations have been carried out by MC. In a first stage, the simulation box is kept fixed, while in a second stage the cross section of the surface is relaxed, releasing the in-plane stress on the slab. The simplicity of the model and the very short range of all interactions allow the extensive sampling of the phase space for a fairly large sample, consisting of 3888 oxygen and 7776 silicon atoms, arranged on a slab exposing two parallel surfaces of $\sim 42 \mathrm{~nm}^{2}$.

Thermodynamic properties computed during the annealing of a liquid sample from $T=2300 \mathrm{~K}$ to $T=0 \mathrm{~K}$ show that the simulated slab undergoes a liquid-to-glass transition at $T_{g}=1660 \pm 50 \mathrm{~K}$, indistinguishable, to within the estimated error bar, from the glass temperature $T_{g}=1680 \pm 50 \mathrm{~K}$ computed for bulk silica. ${ }^{47}$

The surface energy $\left(u_{s}=310 \pm 20 \mathrm{erg} / \mathrm{cm}^{2}\right.$ at $\left.T=300 \mathrm{~K}\right)$ turns out to be surprisingly low, but close to available experimental estimates. ${ }^{11}$ The surface entropy $s_{s}$ has been determined by thermodynamic integration of a polynomial fit to 


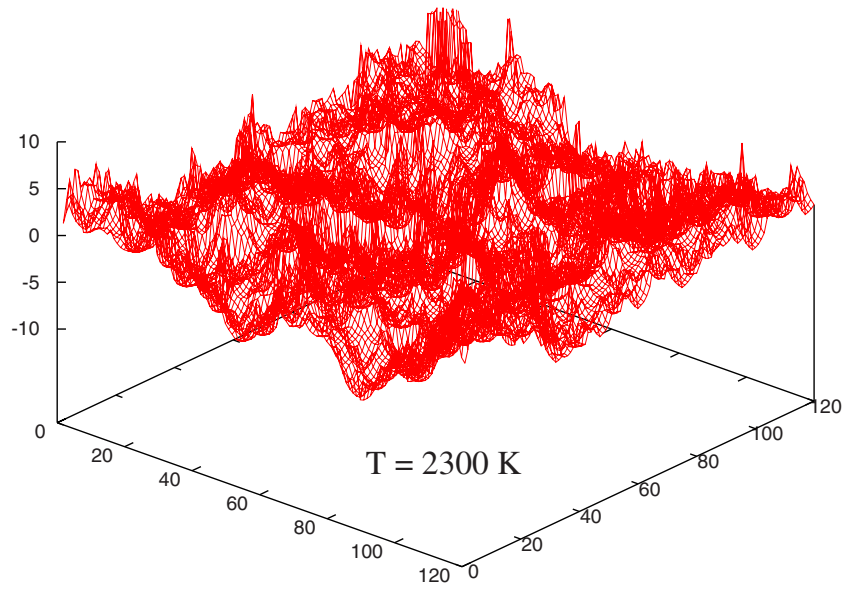

silica and to the larger radius of $\mathrm{O}^{=}$with respect to the radius of $\mathrm{Si}^{4+}$. Relative surface enrichment of oxygen with respect to silicon is observed only at relatively low temperature and in all cases it concerns a thin skin layer no wider than 1-2 A. At $T>1000 \mathrm{~K}$, in fact, a rescaling of the silicon density profile by a constant factor of 2 brings it to practically coincide with the oxygen profile nearly everywhere.

Analysis of the mean-square diffusion of atoms shows that, as expected, in a thin (nearly monoatomic) surface layer, mobility is higher and persists at lower temperatures than in the bulk, pointing to lower diffusion barriers at the surface. This observation of easy surface relaxation is probably related to another important result of our simulation, concerning the roughness of the silica surface. The computation of the height-height correlation function as a function of in-plane distance shows that the liquid surface is rough, i.e., $\langle h(r) h(0)\rangle \sim \log r$ at medium-long distances, while the amorphous silica surface becomes smooth at temperatures below $T_{g}$. The change from rough to smooth observed with decreasing $T$ enhances our confidence on the reliability of the phase-space sampling achieved by our simulations.

The results described and discussed in the present paper show that the idealized force field model of Sec. II augmented by rules to change the bonding topology is flexible and efficient and is able to provide a microscopic view of structural and thermodynamic properties of inhomogeneous silica surfaces. Because of its efficiency, the model could be used to investigate a variety of other inhomogeneous systems, from mesoporous silica to silica clusters.

The model is not easily adapted to molecular-dynamics simulations and therefore dynamical properties are difficult to evaluate. A relation with the time scale can be established via kinetic theory, applied to the activated processes that characterize the real-time dynamics of amorphous systems. Application of this strategy to diffusion in the bulk shows that the time scale of our simulations extends well within the mesoscopic regime and approaches the macroscopic regime, ranging from $0.13 \mathrm{~ms}$ at $T=1800 \mathrm{~K}$ to $0.12 \mathrm{~ms}$ at $T$ $=2000 \mathrm{~K}$. No estimate can be made at lower temperatures, since diffusion is too slow to be detected during our simulations.

The model can be made more realistic by adding an explicit torsion term and possibly other short-range energy contributions such as Urey-Bradley, familiar from classical force fields. On the other hand, the addition of longer-range interactions would spoil the most appealing feature of the present model, i.e., its computational efficiency.

The model can be easily extended to cover other elements, such as hydrogen, germanium, gallium, and phosphorus, covalently bonded to silicon and oxygen. It can also be applied to nonstoichiometric $\mathrm{Si}-\mathrm{SiO}_{2}$ samples, as already done in Ref. 49 within a move-and-minimize scheme.

Finally, the equilibrated slabs produced by our simulations could be exploited for a variety of further investigations concerning surfaces in contact with liquid phases and with a variety of physisorbed molecules, provided the force field is supplemented with appropriate cross interatomic potentials. Atomic positions of well equilibrated samples are available from the authors. 
${ }^{1}$ J. P. Poirier, Introduction to the Physics of the Earth's Interior, 2nd ed. (Cambridge University Press, Cambridge, England, 2000).

${ }^{2}$ F. A. Cotton and G. Wilkinson, Inorganic Chemistry, 5th ed. (Wiley, New York, 1988).

${ }^{3}$ A. P. Legrand, The Surface Properties of Silicas (Wiley, New York, 1998).

${ }^{4}$ V. E. Henrich and P. A. Cox, The Surface Science of Metal Oxides (Cambridge University Press, New York, 1994).

${ }^{5}$ V. Bolis, B. Fubini, L. Marchese, G. Martra, and D. Costa, J. Chem. Soc., Faraday Trans. 87, 497 (1991).

${ }^{6}$ G. J. Young, J. Colloid Interface Sci. 13, 67 (1958).

${ }^{7}$ C. R. Helms and E. H. Poindexter, Rep. Prog. Phys. 57, 791 (1994).

${ }^{8}$ G. Hochstrasser and J. F. Antonini, Surf. Sci. 32, 644 (1972).

${ }^{9}$ C. G. Pantano, Rev. Solid State Sci. 3, 379 (1989).

${ }^{10}$ E. J. Friebele, D. L. Griscomb, and T. W. Hickmott, J. NonCryst. Solids 71, 351 (1985); D. L. Griscom, ibid. 73, 51 (1985).

${ }^{11}$ O. V. Mazurin, M. V. Streltsina, and T. P. ShvaikoShvaikovskaya, Handbook of Glass Data, Part A: Silica Glass and Binary Silicate Glasses (Elsevier, Amsterdam, 1983); J. Mizele, J. L. Dandurand, and J. Schott, Surf. Sci. 162, 830 (1985).

${ }^{12}$ R. B. Laughlin, J. D. Joannopoulos, C. A. Murray, K. J. Hartnett, and T. J. Greytak, Phys. Rev. Lett. 40, 461 (1978).

${ }^{13}$ R. Dupree and R. F. Pettifer, Nature (London) 308, 523 (1984); R. F. Pettifer, R. Dupree, I. Farnan, and U. Sternberg, J. NonCryst. Solids 106, 408 (1988); D. R. Kinney, I.-S. Chuang, and G. E. Maciel, J. Am. Chem. Soc. 115, 6786 (1993).

${ }^{14}$ D. A. Payne, K. S. W. Sing, and D. H. Turk, J. Colloid Interface Sci. 43, 287 (1973).

${ }^{15}$ N. O. Lemcoff and K. S. W. Sing, J. Colloid Interface Sci. 61, 227 (1977).

${ }^{16}$ R. B. Laughlin and J. D. Joannopoulos, Phys. Rev. B 17, 4922 (1978).

${ }^{17}$ V. A. Bakaev, Phys. Rev. B 60, 10723 (1999); V. A. Bakaev and W. A. Steele, J. Chem. Phys. 111, 9803 (1999); V. A. Bakaev, W. A. Steele, T. Bakaeva, and C. G. Pantano, ibid. 111, 9813 (1999).

${ }^{18}$ S. Iarlori, D. Ceresoli, M. Bernasconi, D. Donadio, and M. Parrinello, J. Phys. Chem. B 105, 8007 (2001).

${ }^{19}$ D. Ceresoli, M. Bernasconi, S. Iarlori, M. Parrinello, and E. Tosatti, Phys. Rev. Lett. 84, 3887 (2000).

${ }^{20}$ A. Rimola and P. Ugliengo, J. Chem. Phys. 128, 204702 (2008).

${ }^{21}$ F. Zipoli, D. Donadio, and M. Bernasconi, J. Phys.: Condens. Matter 20, 224011 (2008); F. Zipoli, T. Laino, A. Laio, M. Bernasconi, and M. Parrinello, J. Chem. Phys. 124, 154707 (2006).

${ }^{22}$ L. V. Woodcock, C. A. Angell, and P. Cheeseman, J. Chem. Phys. 65, 1565 (1976).

${ }^{23}$ S. H. Garofalini, J. Chem. Phys. 78, 2069 (1983).

${ }^{24}$ S. H. Garofalini, J. Non-Cryst. Solids 120, 1 (1990).

${ }^{25}$ S. M. Levine and S. H. Garofalini, J. Chem. Phys. 86, 2997 (1987)

${ }^{26}$ B. P. Feuston and S. H. Garofalini, J. Chem. Phys. 91, 564 (1989); J. Appl. Phys. 68, 4830 (1990).

${ }^{27}$ S. Tsuneyuki, M. Tsukada, H. Aoki, and Y. Matsui, Phys. Rev. Lett. 61, 869 (1988).

${ }^{28}$ B. W. H. van Beest, G. J. Kramer, and R. A. van Santen, Phys. Rev. Lett. 64, 1955 (1990).

${ }^{29}$ P. Vashishta, R. K. Kalia, J. P. Rino, and I. Ebbsjö, Phys. Rev. B
41, 12197 (1990); A. Nakano, R. K. Kalia, and P. Vashishta, J. Non-Cryst. Solids 171, 157 (1994); A. Nakano, L. Bi, R. K. Kalia, and P. Vashishta, Phys. Rev. B 49, 9441 (1994).

${ }^{30}$ E. Demiralp, T. Cagin, and W. A. Goddard III, Phys. Rev. Lett. 82, 1708 (1999).

${ }^{31}$ P. Tangney and S. Scandolo, J. Chem. Phys. 117, 8898 (2002).

${ }^{32}$ D. J. Cole, M. C. Payne, G. Csányi, S. M. Spearing, and L. Colombi Ciacchi, J. Chem. Phys. 127, 204704 (2007).

${ }^{33}$ M. I. Trioni, A. Bongiorno, and L. Colombo, J. Non-Cryst. Solids 220, 164 (1997).

${ }^{34}$ A. Roder, W. Kob, and K. Binder, J. Chem. Phys. 114, 7602 (2001).

${ }^{35}$ M. Wilson and T. R. Walsh, J. Chem. Phys. 113, 9180 (2000); T. R. Walsh, M. Wilson, and A. P. Sutton, ibid. 113, 9191 (2000).

${ }^{36}$ N. Mousseau and G. T. Barkema, Curr. Opin. Solid State Mater. Sci. 5, 497 (2001).

${ }^{37}$ C. Mischler, W. Kob, and K. Binder, Comput. Phys. Commun. 147, 222 (2002).

${ }^{38}$ See, for instance, the $a b$ initio simulation of the hydroxilated silica surface in F. Tielens, C. Gervais, J.-F. Lambert, F. Mauri, and D. Costa, Chem. Mater. 20, 3336 (2008).

${ }^{39}$ P. N. Keating, Phys. Rev. 145, 637 (1966).

${ }^{40}$ F. Wooten, K. Winer, and D. Weaire, Phys. Rev. Lett. 54, 1392 (1985); F. Wooten and D. Weaire, Solid State Phys. 40, 1 (1987).

${ }^{41}$ R. L. C. Vink and G. T. Barkema, Phys. Rev. B 67, 245201 (2003).

${ }^{42}$ S. von Alfthan, A. Kuronen, and K. Kaski, Phys. Rev. B 68, 073203 (2003).

${ }^{43}$ D. I. Grimley, A. C. Wright, and R. N. Sinclair, J. Non-Cryst. Solids 119, 49 (1990); A. C. Wright, R. A. Hulme, D. I. Grimley, R. N. Sinclair, S. W. Martin, D. L. Price, and F. L. Galeener, ibid. 129, 213 (1991).

${ }^{44}$ S. Susman, K. J. Volin, D. L. Price, M. Grimsditch, J. P. Rino, R. K. Kalia, P. Vashishta, G. Gwanmesia, Y. Wang, and R. C. Liebermann, Phys. Rev. B 43, 1194 (1991).

${ }^{45}$ R. L. C. Vink and G. T. Barkema, Phys. Rev. Lett. 89, 076405 (2002).

${ }^{46}$ Y.-H. Tu, J. Tersoff, G. Grinstein, and D. Vanderbilt, Phys. Rev. Lett. 81, 4899 (1998).

${ }^{47}$ R. Cabriolu, M. G. Del Pópolo, and P. Ballone, Phys. Chem. Chem. Phys. 11, 10820 (2009).

${ }^{48}$ E. Bourova and P. Richet, Geophys. Res. Lett. 25, 2333 (1998); L. Huang and J. Kieffer, Phys. Rev. Lett. 95, 215901 (2005).

${ }^{49}$ Y.-H. Tu and J. Tersoff, Phys. Rev. Lett. 84, 4393 (2000).

${ }^{50}$ S. L. Chan and S. R. Elliott, J. Phys.: Condens. Matter 4, 1269 (1992).

${ }^{51}$ A. F. Wright and A. J. Leadbetter, Philos. Mag. 31, 1391 (1975).

${ }^{52}$ S. Coh and D. Vanderbilt, Phys. Rev. B 78, 054117 (2008).

${ }^{53}$ H. Kimizuka, H. Kaburaki, and Y. Kogure, Phys. Rev. Lett. 84, 5548 (2000).

${ }^{54}$ A. Polian, D. Vo-Thanh, and P. Richet, Europhys. Lett. 57, 375 (2002).

${ }^{55}$ B. A. Auld, Acoustic Fields and Waves in Solids (Wiley, New York, 1973), Vol. 1, p. 368.

${ }^{56}$ V. G. Zubov and M. M. Firsova, Kristallografiya 7, 469 (1962) [Sov. Phys. Crystallogr. 7, 374 (1962)]; I. Ohno, J. Phys. Earth 43, 157 (1995).

${ }^{57}$ G. T. Barkema and N. Mousseau, Phys. Rev. Lett. 77, 4358 
(1996); N. Mousseau and G. T. Barkema, Phys. Rev. E 57, 2419 (1998).

${ }^{58}$ P. Richet, Y. Bottinga, L. Denielou, J. P. Petitet, and C. Tequi, Geochim. Cosmochim. Acta 46, 2639 (1982).

${ }^{59}$ G. Brebec, R. Seguin, C. Sella, J. Bevenot, and J. C. Martin, Acta Metall. 28, 327 (1980).

${ }^{60}$ H. F. Wolf, Semiconductors (Wiley Interscience, New York, 1971), p. 21.

${ }^{61}$ A. Zangwill, Physics at Surfaces (Cambridge University Press,
Cambridge, England, 1988).

${ }^{62}$ J. D. Stevenson and P. G. Wolynes, J. Chem. Phys. 129, 234514 (2008).

${ }^{63}$ P. Ballone and S. Rubini, Phys. Rev. Lett. 77, 3169 (1996); Surf. Sci. 342, L1116 (1995).

${ }^{64}$ C. Vree and S. G. Mayr, New J. Phys. 12, 023001 (2010).

${ }^{65}$ J. Toner and D. P. DiVincenzo, Phys. Rev. B 41, 632 (1990); S. Scheidl, Phys. Rev. Lett. 75, 4760 (1995); D. Cule and Y. Shapir, ibid. 74, 114 (1995). 